\title{
A non-canonical function for Centromere-associated protein-E (CENP-E) controls centrosome integrity and orientation of cell division
}

\author{
Mikito Owa ${ }^{*}$ and Brian Dynlacht ${ }^{*}$
}

1. Department of Pathology, New York University Cancer Institute, New York University School of Medicine, New York, NY, 10016, USA

Correspondence: Mikito.Owa@nyumc.org, Brian.Dynlacht@nyumc.org 


\section{Summary}

2 Centromere-associated protein-E (CENP-E) is a kinesin motor localizing at kinetochores.

3 Although its mitotic functions have been well studied, it has been challenging to investigate

4 direct consequences of CENP-E removal using conventional methods because CENP-E

5 depletion results in mitotic arrest. In this study, we harnessed an auxin-inducible degron

6 system to achieve acute degradation of CENP-E. We revealed a kinetochore-independent role

7 for CENP-E that removes pericentriolar material 1 (PCM1) from centrosomes in $\mathrm{G}_{2}$ phase.

8 After acute loss of CENP-E, centrosomal Polo-like kinase 1 (Plk1) is sequestered by

9 accumulated PCM1, resulting in aberrant phosphorylation and destabilization of centrosomes,

10 which triggers loss of astral microtubules and oblique cell divisions. Furthermore, we also

11 observed centrosome and cell division defects in cells from a microcephaly patient with

12 mutations in CENPE. Orientation of cell division is deregulated in some microcephalic

13 patients, and our unanticipated findings provide a unifying principle that explains how

14 microcephaly can result from centrosomal defects. 


\section{Introduction}

The centrosome is an organizing center for microtubules (MTs) in metazoan

18 cells. This organelle functions as a hub for MT-based protein transport during interphase, and

19 it organizes spindle poles in mitosis. Within the centrosome, the two centrioles are embedded

20 in pericentriolar material (PCM), which consists of $\gamma$-tubulin ring complexes, a nucleator of

21 MTs, together with a cohort of proteins that regulate centrosome integrity ${ }^{1}$. Centrosomes and

22 the environment surrounding this organelle are dynamically remodeled throughout the cell

23 cycle. In interphase, centriolar satellites, dense granules that consist of multiple proteins

24 essential for controlling centriole duplication, are scattered around centrosomes ${ }^{2,3}$. These

25 granules are anchored on microtubules (MTs) through a scaffold protein, pericentriolar

26 material 1 (PCM1; despite its name, this is not a component of the PCM). Beyond its ability

27 to organize centriolar satellites on the MTs, PCM1 also prevents its interacting partners from

28 being inappropriately relocated to centrosomes, as suggested by the observation that satellite

29 proteins are constitutively localized at centrosomes after PCM1 depletion ${ }^{4-8}$. Centriolar

30 satellites accumulate around centrosomes through dynein-driven transport during and after

31 centriole duplication ${ }^{9-11}$. Subsequently, they are re-distributed within the cytoplasm as

32 prophase commences ${ }^{4,5}$. On the other hand, Plk1 accumulates on the centrosome in prophase,

33 where it phosphorylates PCM components, including pericentrin (PCNT) and Wdr62 $2^{12,13}$.

34 These modifications accelerate PCM expansion and formation of robust astral MTs, which

35 anchor the cell cortex to maintain spindle orientation. Despite our knowledge of these

36 dynamic events during prophase, the mechanisms underlying the dispersal of centriolar

37 satellites - and its functional implications - remain obscure, and how PCM expansion is

38 spatio-temporally regulated likewise remains unclear. 
Recent proteomic studies have identified motor proteins that potentially interact with

40 centriolar satellites $^{14,15}$, and centromere-associated protein-E (CENP-E) is one such

41 candidate. CENP-E is a kinesin motor localizing to kinetochores, where the protein reinforces

42 MT-kinetochore interactions ${ }^{16-20}$, and contributes to the spindle assembly checkpoint ${ }^{21-23}$.

43 CENP-E depletion in mammals leads to mitotic arrest ${ }^{21,22}$, and deletion of CENPE in mice

44 results in early embryonic lethality ${ }^{24}$. However, all known functions for CENP-E pertain to

45 mitosis, during which time centriolar satellites are dispersed in the cytoplasm. Therefore, the

46 functional consequences for interactions between CENP-E and centriolar satellite proteins

47 remain elusive. In this study, we show that CENP-E has a non-canonical role around

48 centrosomes in interphase. CENP-E removes PCM1 from the peri-centrosomal region in $\mathrm{G}_{2}$

49 phase, and this transport is critical for structural stability of centrosomes and maintenance of

50 spindle orientation in mitosis. Moreover, our findings can explain phenotypes associated with

51 microcephaly, a developmentally-related brain disorder, using patient-derived cells mutated

52 in $C E N P E^{25}$. Thus, we have unveiled an unanticipated role for CENP-E in centrosome

53 dynamics and have linked it to mechanisms that result in microcephaly.

$58 \quad$ Results

\section{CENP-E is recruited around centrosomes in $G_{2}$ phase}

It was previously reported that CENP-E protein is initially synthesized prior to

61 mitosis $^{16}$. Western blotting of lysates from synchronized wild-type RPE-1 cells confirmed

62 that cytoplasmic CENP-E levels were elevated in late S/G2 phase, and they peaked in mitosis 
63 (Fig. 1a). However, in late S/G2 phase, MTs are not attached to kinetochores, and therefore,

64 whether cytoplasmic CENP-E had a function in interphase was unclear. To understand its

65 interphase role, we first explored CENP-E localization from interphase to mitotic onset using

66 immunofluorescence. In early S phase, CENP-E signal was not detectable in the cytoplasm,

67 in accordance with its low expression levels (Fig. 1b,c, 0 and $6 \mathrm{~h}$ ). On the other hand,

68 CENP-E was specifically enriched around centrosomes from late S to G2 phase (Fig. 1b,c, 10

69 and 12h), and was dispersed throughout the cytoplasm by prophase (Fig. 1b,c, prophase).

70 This pericentrosomal localization of CENP-E is consistent with recently identified

71 interactions between centriolar satellites, typified by the PCM1 protein, and CENP-E ${ }^{15}$.

72 Given that centriolar satellites are not localized at kinetochores ${ }^{4,5}$, these data suggest that

73 CENP-E has a previously uncharacterized role around the centrosome in interphase.

\section{Loss of CENP-E leads to cell cycle exit after one cell division cycle}

CENP-E depletion using siRNA or antibody injection leads to mitotic arrest $\mathrm{t}^{17,20,22}$.

77 However, these methods require extended periods of time for complete depletion or inactivation and depend on the specificity of siRNAs or antibodies. Therefore, it has been challenging to study the immediate consequences of CENP-E removal. To overcome these

80 problems and investigate the peri-centrosomal function of CENP-E, we established a

81 conditional knockout $(\mathrm{KO})$ cell line using gene-editing to biallelically introduce

82 Auxin-inducible degrons at the endogenous locus (CENP-E-AID) ${ }^{26-29}$. One hour after auxin

83 (IAA) addition, CENP-E was degraded and undetectable by western blotting (Fig. 2a).

84 Further, CENP-E signal was not detectable around centrosomes in interphase or at

85 kinetochores in mitosis upon auxin treatment (Fig. 2b). Most mitotic CENP-E KO cells had

86 misaligned chromosomes near spindle poles, a phenotype consistent with other CENP-E

87 depletion studies ${ }^{17,20,22,23,30}$ (Fig. 2b arrowheads; Extended Data Fig. 2a). In agreement with 
88 a previous report in mice ${ }^{23}$, the complete loss of CENP-E did not lead to mitotic arrest but

89 instead prompted a delay in metaphase (Fig. 2c). Importantly, nearly all CENP-E KO cells

90 stopped growing and exited from the cell cycle after one cell division cycle (Fig. 2d,e;

91 Extended Data Fig. 1b). Loss of CENP-E led to chromosome mis-segregation in mice ${ }^{23}$,

92 suggesting that our CENP-E KO cells might exit from the cell cycle due to aneuploidy in

93 daughter cells.

\section{Loss of CENP-E promotes PCM fragmentation and oblique cell divisions}

A previous study reported that spindle MTs were not properly focused at spindle poles in a small fraction of cells treated with CENP-E siRNA, despite exclusive localization of CENP-E at kinetochores in wild-type metaphase cells ${ }^{22}$. Focusing of spindle MTs at poles is dependent on dynein-driven transport along astral MTs, nucleated at the $\mathrm{PCM}^{31}$. Therefore,

100 the possibility remained that unfocused spindle MTs in CENP-E-depleted cells arose from

101 defects in PCM structure. Interestingly, by immuno-staining for PCM markers, we showed

102 that loss of CENP-E also provoked severe defects in PCM morphology. In controls without 103 auxin treatment, $\gamma$-tubulin and PCNT were sharply focused at each spindle pole (Fig. 3a,

104 -IAA). On the other hand, $\gamma$-tubulin and PCNT foci were fragmented in 55\% of CENP-E KO

105 cells upon auxin addition (Fig. 3a, +IAA). Furthermore, cells treated with GSK923295 $106(\mathrm{GSK})^{32}$, an inhibitor of CENP-E motor activity, also showed PCM fragmentation (77\%; Fig.

107 3b), as well as misaligned chromosomes (Extended Data Fig. 2b), suggesting that 108 fragmentation in CENP-E KO cells results from loss of CENP-E-dependent protein transport.

109 Although a recent study suggested that prolonged prometaphase induced by drug treatments

110 led to precocious centriole disengagement and PCM fragmentation in a separase-dependent

111 manner $^{33}$, we did not observe a significant increase in separated centrioles after GSK

112 treatment (Extended Data Fig. 3a). Instead, centrioles were misaligned with respect to the 
113 equator in $\sim 16 \%$ of metaphase CENP-E KO cells (Extended Data Fig. 3b). These results

114 suggest that PCM fragmentation in the CENP-E KO was not driven by enhanced separase

115 activity, but rather that PCM was detached from centrioles.

116 Strikingly, we also found that $\sim 44 \%$ of mitotic CENP-E KO cells lost astral MTs, 117 consistent with PCM fragmentation in those cells (Fig. 3c). To further investigate this 118 phenotype, we performed live-cell imaging of cells with and without auxin treatment. The

119 appearance of abnormal astral MTs in the CENP-E KO resulted in a high proportion of cells 120 undergoing rotation of their metaphase plates ( $\sim 50 \%$; Fig. 3d,e; Supplementary Movie 1) and 121 an oblique cell division angle ( $41 \%$; Fig. 3 d,f). Intriguingly, these phenotypes are

122 reminiscent of knock-down of $A S P M$ and $W d r 62$, two genes implicated in microcephaly that 123 encode mitotic spindle and centrosome proteins ${ }^{13,34,35}$. CENPE mutations have also been 124 implicated in microcephaly ${ }^{25}$, and our results imply that analogous mechanisms could link the 125 disease phenotypes arising from these three mutations.

\section{PCM fragmentation in CENP-E KO is linked to aberrant Plk1 activity}

128 Since centrosomal Plk1 phosphorylates PCM components in prophase, and this 129 phosphorylation is essential for robust PCM expansion ${ }^{1,}{ }^{12}$, it was possible that PCM 130 fragmentation in CENP-E KO reflected an aberrant modification of its constituents. To test 131 this possibility, we first explored centrosomal Plk1 levels in prophase CENP-E-AID cells. By

132 immunofluorescence, we revealed that centrosomal Plk1 levels in CENP-E KO cells were 133 significantly diminished as compared to controls (Fig. 4a,b). We next investigated the impact 134 of this Plk1 reduction on PCNT, as it is a key substrate and an essential factor for PCM 135 expansion $^{12}$. Previous studies have identified sites on PCNT phosphorylated by Plk1, 136 including S1241 ${ }^{12}$, which is essential for PCM expansion, and multiple carboxy-terminal sites 137 around $\mathrm{R} 2331^{36}$ that are essential for PCNT cleavage during mitotic exit. After 
138 immuno-staining with a phospho-specific antibody (PCNT-pS1241) ${ }^{12}$, we found that the

139 fluorescence intensity of pS1241 was significantly lower on centrosomes in prophase

140 CENP-E KO cells as compared to controls, similar to our findings with Plk1 (Fig. 4c.d). We

141 further tested PCNT cleavage to examine the C-terminal phosphorylation of PCNT.

142 Treatment with ZM447439 (ZM, an inhibitor of Aurora kinases) results in forced exit of

143 mitotically arrested cells from M phase, and PCNT is cleaved in a Plk1-phosphorylation

144 dependent manner ${ }^{36}$. Although we did not detect the cleaved form of PCNT, most likely due

145 to rapid degradation after cleavage, full-length PCNT was nearly undetectable in ZM-treated

146 controls replete with CENP-E, suggesting near-complete cleavage during mitotic exit (Fig. 4e,

147 -IAA). As a control, and as described previously ${ }^{36}$, we showed that this cleavage was

148 substantially blocked by inhibition of Plk1 through BI2536 (BI) treatment, attesting to its

149 dependence on Plk1 (Fig. 4e, compare lanes 2 and 6). Importantly, similar to Plk1-inhibited

150 cells, ZM-treated CENP-E KO cells retained full-length PCNT, at levels approximating those

151 in untreated cells (Fig. 4e, +IAA; compare lanes 3 and 4). Thus, we conclude that Plk1 fails

152 to efficiently phosphorylate PCNT in CENP-E KO cells.

153 Finally, to determine whether the reduction in Plk1 levels could explain the PCM

154 defects, we ectopically expressed Plk1 in CENP-E KO cells. Notably, over-expression of

155 constitutively active Plk1 (Plk1-T210D) in CENP-E KO rescued PCM fragmentation,

156 whereas expression of the catalytically inactive protein (Plk1-K82R) did not (Fig. 4f,g). In

157 contrast, chromosome misalignment was not rescued by over-expression of P1k1-T210D

158 (Extended Data Fig. 2c). These data strongly suggest that (1) the reduction of Plk1 levels on

159 centrosomes in CENP-E KO cells results in PCM fragmentation and (2) PCM defects in

160 CENP-E KO cells are independent of kinetochore dysfunction associated with chromosome

161 misalignment. 
165 dispersed by prophase, during which time centriolar satellites are re-distributed ${ }^{4}$. Since

166 CENP-E is a processive kinesin that moves toward the plus end of MTs ${ }^{17}$, we hypothesized

167 that CENP-E transports PCM1 from the vicinity of centrosomes, as it is redistributed 168 throughout the cytoplasm by the initiation of prophase. We therefore investigated the 169 distribution of PCM1 around centrosomes in prophase cells. In control cells, PCM1 exhibited 170 the expected centriolar satellite-like localization, with PCM1 foci scattered around the 171 centrosome (Fig. 5a, -IAA). In contrast, PCM1 was tightly concentrated within the immediate 172 vicinity of centrosomes in CENP-E KO cells (Fig. 5a, +IAA; Fig. 5b). These data suggest 173 that CENP-E removes PCM1 from the peri-centrosomal region by prophase, during which 174 time Plk1 phosphorylates PCM proteins.

As described above, a recent proteome-wide study identified interactions between

176 CENP-E and PCM1, although this interaction was not explored in detail ${ }^{15}$. To determine

177 whether these interactions reflected the observed CENP-E-dependent PCM1 redistribution 178 and to clarify the cell cycle stage in which CENP-E interacts with PCM1, we performed 179 immunoprecipitations with lysates from CENP-E-AID cells synchronized by treatment with 180 mimosine (late $\mathrm{G}_{1}$ phase) or monastrol (M phase). PCM1 co-precipitated with CENP-E in 181 samples released from mimosine for 6 and 10 hours (Fig. 5c, $6 \mathrm{~h}$ and 10h), and the amount of 182 co-precipitated PCM1 increased in accordance with CENP-E levels. On the other hand, 183 PCM1 was not detected in precipitates from lysates of cells released for 12 hours (Fig. 5c, 184 12h) or from monastrol-synchronized cells (Fig. 5c, M). Given that most cells reach $\mathrm{G}_{2}$ phase 185 by $10 \mathrm{~h}$ after release from mimosine (Extended Data Fig. 4), these data demonstrate that 186 CENP-E interacts with PCM1 most likely in late $\mathrm{S} / \mathrm{G}_{2}$ phase, consistent with the idea that 187 CENP-E transports PCM1 and centriolar satellites during this period. 


\section{PCM1 depletion rescues PCM-related defects in CENP-E KO}

In CENP-E KO cells, peri-centrosomal PCM1 accumulation coincided with Plk1

191 reduction at centrosomes in prophase (Fig. 4a and Fig. 5a). Since PCM1 is thought to restrict

192 centriolar satellite proteins from being recruited to centrosomes ${ }^{4-8}$, it was conceivable that

193 PCM1 aberrantly sequestered Plk1 from centrosomes in KO cells. Therefore, we performed

194 PCM1 ablation experiments in CENP-E-AID cells to examine whether PCM1 that

195 concentrated in CENP-E KO cells (Fig. 5a,b) physically sequesters Plk1 from centrosomes.

196 With control siRNA treatment, centrosomal intensity of Plk1 in prophase CENP-E KO cells

197 was reduced as expected (Fig. 6a,b, Cntrl; compare with Fig. 4a,b). In striking contrast, loss

198 of CENP-E did not result in a reduction in centrosomal Plk1 in PCM1-depleted cells (Fig.

199 6a,b, siPCM1), suggesting that the accumulation of PCM1 in CENP-E KO cells is directly 200 responsible for perturbing Plk1 recruitment to centrosomes.

201 Importantly, and consistent with the recovery of centrosomal Plk1 levels, siPCM1 202 treatment reversed the reduction in PCNT phosphorylation at S1241 observed in CENP-E 203 KO cells (Fig. 6c,d). Consequently, PCM1 depletion also rescued both PCM fragmentation 204 (Fig. 6e) and the appearance of aberrant astral MTs caused by the loss of CENP-E (Fig. 6f). 205 These data strongly suggest that the PCM-related phenotypes in CENP-E KO cells stem from 206 the accumulation of PCM1 around centrosomes triggered by the absence of a motor protein 207 that normally moves it away from the organelle. Importantly, chromosome misalignment in 208 CENP-E KO was not rescued by PCM1 depletion (Extended Data Fig. 2d), indicating that the 209 PCM1-Plk1 pathway is not involved in kinetochore defects caused by the loss of CENP-E.

211 Patient LCLs mutated in CENPE exhibit PCM and cell division defects 
214 within the coiled-coil region in the middle of the protein but do not affect CENPE expression

215 levels. Moreover, as the mutations did not result in embryonic lethality, lymphoblastoid cell

216 lines (LCLs) derived from these patients have been successfully established, providing

217 further evidence that disease mutations in CENPE do not lead to cell cycle exit, in contrast

218 with our CENP-E KO cells. To examine whether the mutations drive PCM-related defects

219 observed in the CENP-E KO, we explored PCM structures by immunofluorescence using

220 LCLs from one of the patients and an apparently healthy donor (wild-type). Although PCM

221 fragmentation was rarely observed in the patient LCLs, centrosomal PCNT levels in 222 metaphase were significantly decreased compared to wild-type cells (Fig. 7a.b), suggesting

223 that the mutations in CENPE affect PCM expansion and/or maintenance of PCM structures.

224 Consistently, centrosomal p1241 levels also significantly decreased in prophase patient cells

225 (Fig. 7c,d). In agreement with the morphological defects in patient PCM, live-cell imaging 226 revealed that chromosomes were obliquely segregated into daughter cells in $41 \%$ of patient

227 LCLs, whereas the percentage of oblique cell divisions in wild-type cells was $13 \%$ (Fig. 7e,f).

228 These data indicate that the heterozygous mutations in CENPE in microcephaly patients lead 229 to PCM defects resulting in oblique cell divisions, which is a typical phenotype associated 230 with the disease. 


\section{Discussion}

CENP-E is a kinesin motor that transports chromosomes to the metaphase plate along

234 spindle MTs. Persistent, complete loss of CENP-E causes delays in metaphase, most likely

235 due to incomplete chromosome alignment, followed by cell cycle exit (Fig. 2c-e; Extended

236 Data Fig. 1b) $)^{22,23}$. Therefore, it has been challenging to investigate direct consequences of

237 CENP-E removal using siRNA or conventional KO cell lines. Here, acute degradation of

238 CENP-E in our AID cell line enabled us to identify and characterize a second, distinct

239 function for CENP-E apart from its role at kinetochores (Fig. 7g). Namely, we found that in

$240 \mathrm{G}_{2}$ phase, CENP-E transports PCM1 from the vicinity of centrosomes to a location occupied

241 by centriolar satellites. This redistribution of PCM1 is important to maintain the level of

242 centrosomal Plk1 in prophase, when it phosphorylates PCNT to facilitate PCM expansion ${ }^{12,36}$.

243 Loss of CENP-E leads to reductions in phosphorylation levels of PCM, resulting in PCM

244 fragmentation, loss of astral MTs, and oblique cell divisions. We propose that CENP-E

245 represents the first kinesin identified as a transporter of centriolar satellites, moving them

246 away from the centrosome. However, we note that $\sim 50 \%$ of CENP-E KO cells retained a

247 normal PCM appearance (Fig. 3a), implying that another kinesin or MT remodeling in

248 mitosis could also contribute to the redistribution of PCM1 cooperatively or redundantly with

249 CENP-E.

A recent study reported that centromere dysfunction causes dispersion of PCM during

251 mitosis $^{37}$. Since CENP-E is a component of the kinetochore, it was possible that this effect

252 may partially overlap phenotypes caused by the loss of CENP-E. However, the dispersion

253 under kinetochore dysfunction was limited to the vicinity of spindle poles, in contrast to PCM

254 fragmentation caused by loss of CENP-E or its inhibition (Fig. 3a,b). Furthermore, the

255 PCM-related phenotypes observed in CENP-E KO cells were rescued by over-expression of

256 active-Plk1 or PCM1 depletion (Fig. 4f,g; Fig. 6), whereas chromosome misalignment, a 
phenotype induced by kinetochore dysfunction, was not rescued through these manipulations

258 (Extended Data Fig. 2c.d). These findings indicate that the second, interphase function for

259 CENP-E is functionally separable from its role at kinetochores, and the interphase pathway

260 we propose in our model is most critical for maintenance of PCM integrity during mitosis.

261

Despite PCM fragmentation and loss of astral MTs after CENP-E removal, most of

263 our KO cells formed bipolar spindles. Since astral MTs are essential for bipolar spindle

264 formation $^{31}$, our results suggest that PCM in CENP-E KO cells is fragmented by MT-induced

265 forces between the cortex or chromosomes and centrosomes after bipolar spindle formation.

266 Indeed, live-cell imaging revealed that all of the CENP-E KO cells formed a metaphase plate

267 after NEBD (Fig. 3d; Supplementary Movie 1). Furthermore, the fragmented PCM in

268 CENP-E KO cells did not capture chromosomes, suggesting that the PCM foci did not

269 ectopically accumulate but that they represent PCM that has dissociated from centrosomes

270 after bipolar spindle formation due to aberrant phosphorylation of its components. Spindle

271 MTs attached to kinetochores are cross-bridged to each other by a clathrin-mediated

272 complex $^{38,39}$. Thus, we speculate that once bipolar spindles are formed, KO cells are able to

273 maintain bipolarity even if the focused ends of the fibers are detached from spindle poles. On

274 the other hand, astral MTs are the only identified structures that connect spindle poles with

275 the cell cortex. Loss of astral MTs in CENP-E KO cells therefore leads to release of the

276 bipolar spindles from the cortex, resulting in rotation of metaphase plates and oblique cell 277 divisions.

To date, 25 genes have been implicated in autosomal recessive primary microcephaly

279 (MCPH; OMIM phenotypic series: PS251200) ${ }^{35,40}$, and CENPE (MCPH13) is one of these 280 genes. Although PCNT is not included among the MCPH genes, mutations in the gene cause 281 microcephalic osteodysplastic primordial dwarfism type II (MOPDII) ${ }^{41-43}$, suggesting strong 
correlations between PCM defects and microcephaly. Since CENP-E was first identified as a

283 component of the kinetochore involved in chromosome alignment, it was anticipated that the

284 mutations in CENPE provoke microcephaly through defects in chromosome segregation.

285 However, CENP-E patient cells do not exhibit cell cycle exit seen in our CENP-E KO, 286 suggesting that CENP-E mutant proteins in patients retain partial activity essential for cell 287 cycle progression. These data therefore suggest a new mechanism for diseases associated 288 with CENP-E mutations. Using patient LCLs, we revealed that the mutations lead to 289 reductions in the mass of PCM and oblique cell divisions. ASPM (MCPH5) and Wdr62 290 (MCPH2) also enforce proper spindle orientation through astral MT organization ${ }^{13,34}$, and 291 our findings suggest a generalized mechanism for control of cell division orientation, which 292 is de-regulated during brain development in some microcephalic patients.

294 interphase CENP-E--separable from its role at kinetochores--that is critical for maintenance 295 of PCM structure, astral MTs, and spindle orientation, akin to the function of other genes 296 mutated in microcephaly. 


\section{References}

299 1. Woodruff, J.B., Wueseke, O. \& Hyman, A.A. Pericentriolar material $300 \quad$ structure and dynamics. Philos Trans $R$ Soc Lond B Biol Sci 369 (2014).

301 2. Hori, A. \& Toda, T. Regulation of centriolar satellite integrity and its $302 \quad$ physiology. Cell Mol Life Sci 74, 213-229 (2017).

303 3. Prosser, S.L. \& Pelletier, L. Centriolar satellite biogenesis and function in $304 \quad$ vertebrate cells. J Cell Sci 133 (2020).

3054 4. Sedjai, F. et al. Control of ciliogenesis by FOR20, a novel centrosome and 306

307 5. Lopes, C.A. et al. Centriolar satellites are assembly points for proteins 308 implicated in human ciliopathies, including oral-facial-digital syndrome 1.

310 6. Kim, K., Lee, K. \& Rhee, K. CEP90 is required for the assembly and centrosomal accumulation of centriolar satellites, which is essential for primary cilia formation. PLoS One 7, e48196 (2012).

313 7. Stowe, T.R., Wilkinson, C.J., Iqbal, A. \& Stearns, T. The centriolar satellite proteins Cep72 and Cep290 interact and are required for recruitment of BBS proteins to the cilium. Mol Biol Cell 23, 3322-3335 (2012).

316 8. Wang, L., Lee, K., Malonis, R., Sanchez, I. \& Dynlacht, B.D. Tethering of 317 an E3 ligase by PCM1 regulates the abundance of centrosomal KIAA0586/Talpid3 and promotes ciliogenesis. Elife 5 (2016).

319 9. Kubo, A., Sasaki, H., Yuba-Kubo, A., Tsukita, S. \& Shiina, N. Centriolar 320 satellites: molecular characterization, ATP-dependent movement toward 
centrioles and possible involvement in ciliogenesis. J Cell Biol 147, 969-980 (1999).

323

324

325

326

327

328

329

330

331

332

333

334

335

336

337

338

339

340

341

342

343

344

10. Dammermann, A. \& Merdes, A. Assembly of centrosomal proteins and microtubule organization depends on PCM-1. J Cell Biol 159, 255-266 (2002).

11. Kim, J.C. et al. The Bardet-Biedl protein BBS4 targets cargo to the pericentriolar region and is required for microtubule anchoring and cell cycle progression. Nat Genet 36, 462-470 (2004).

12. Lee, K. \& Rhee, K. PLK1 phosphorylation of pericentrin initiates centrosome maturation at the onset of mitosis. J Cell Biol 195, 1093-1101 (2011).

13. Miyamoto, T. et al. PLK1-mediated phosphorylation of WDR62/MCPH2 ensures proper mitotic spindle orientation. Hum Mol Genet 26, 4429-4440 (2017).

14. Gupta, G.D. et al. A Dynamic Protein Interaction Landscape of the Human Centrosome-Cilium Interface. Cell 163, 1484-1499 (2015).

15. Hein, M.Y. et al. A human interactome in three quantitative dimensions organized by stoichiometries and abundances. Cell 163, 712-723 (2015).

16. Yen, T.J., Li, G., Schaar, B.T., Szilak, I. \& Cleveland, D.W. CENP-E is a putative kinetochore motor that accumulates just before mitosis. Nature $359,536-539$ (1992).

17. Wood, K.W., Sakowicz, R., Goldstein, L.S. \& Cleveland, D.W. CENP-E is a plus end-directed kinetochore motor required for metaphase chromosome alignment. Cell 91, 357-366 (1997). 
345 18. Yao, X., Anderson, K.L. \& Cleveland, D.W. The microtubule-dependent motor centromere-associated protein E (CENP-E) is an integral component of kinetochore corona fibers that link centromeres to spindle microtubules. J Cell Biol 139, 435-447 (1997).

19. Cooke, C.A., Schaar, B., Yen, T.J. \& Earnshaw, W.C. Localization of CENP-E in the fibrous corona and outer plate of mammalian kinetochores from prometaphase through anaphase. Chromosoma 106, 446-455 (1997).

352 20. Schaar, B.T., Chan, G.K., Maddox, P., Salmon, E.D. \& Yen, T.J. CENP-E 353 function at kinetochores is essential for chromosome alignment. J Cell Biol 139, 1373-1382 (1997).

21. Abrieu, A., Kahana, J.A., Wood, K.W. \& Cleveland, D.W. CENP-E as an essential component of the mitotic checkpoint in vitro. Cell 102, 817-826 (2000).

22. Yao, X., Abrieu, A., Zheng, Y., Sullivan, K.F. \& Cleveland, D.W. CENP-E forms a link between attachment of spindle microtubules to kinetochores and the mitotic checkpoint. Nat Cell Biol 2, 484-491 (2000).

361 23. Weaver, B.A. et al. Centromere-associated protein-E is essential for the mammalian mitotic checkpoint to prevent aneuploidy due to single chromosome loss. J Cell Biol 162, 551-563 (2003).

364 24. Putkey, F.R. et al. Unstable kinetochore-microtubule capture and chromosomal instability following deletion of CENP-E. Dev Cell 3, 351-365 (2002). 
367 25. Mirzaa, G.M. et al. Mutations in CENPE define a novel kinetochore-centromeric mechanism for microcephalic primordial dwarfism. Hum Genet 133, 1023-1039 (2014).

26. Nishimura, K., Fukagawa, T., Takisawa, H., Kakimoto, T. \& Kanemaki, M. An auxin-based degron system for the rapid depletion of proteins in nonplant cells. Nat Methods 6, 917-922 (2009).

27. Samejima, K. et al. Auxin-induced rapid degradation of inhibitor of caspase-activated DNase (ICAD) induces apoptotic DNA fragmentation, caspase activation, and cell death: a cell suicide module. J Biol Chem 289, 31617-31623 (2014).

28. Natsume, T., Kiyomitsu, T., Saga, Y. \& Kanemaki, M.T. Rapid Protein Homology Donors. Cell Rep 15, 210-218 (2016).

29. Yesbolatova, A., Natsume, T., Hayashi, K.I. \& Kanemaki, M.T. Generation of conditional auxin-inducible degron (AID) cells and tight control of degron-fused proteins using the degradation inhibitor auxinole. Methods 164-165, 73-80 (2019).

384 30. McEwen, B.F. et al. CENP-E is essential for reliable bioriented spindle 385 attachment, but chromosome alignment can be achieved via redundant mechanisms in mammalian cells. Mol Biol Cell 12, 2776-2789 (2001).

387 31. Walczak, C.E., Vernos, I., Mitchison, T.J., Karsenti, E. \& Heald, R. A 388 model for the proposed roles of different microtubule-based motor proteins in establishing spindle bipolarity. Curr Biol 8, 903-913 (1998). 
390 32. Wood, K.W. et al. Antitumor activity of an allosteric inhibitor of centromere-associated protein-E. Proc Natl Acad Sci U S A 107, 5839-5844 (2010).

393 33. Karki, M., Keyhaninejad, N. \& Shuster, C.B. Precocious centriole disengagement and centrosome fragmentation induced by mitotic delay. Nat Commun 8, 15803 (2017).

34. Gai, M. et al. ASPM and CITK regulate spindle orientation by affecting the dynamics of astral microtubules. EMBO Rep 17, 1396-1409 (2016).

35. Jayaraman, D., Bae, B.I. \& Walsh, C.A. The Genetics of Primary Microcephaly. Annu Rev Genomics Hum Genet 19, 177-200 (2018).

400 36. Kim, J., Lee, K. \& Rhee, K. PLK1 regulation of PCNT cleavage ensures 401 fidelity of centriole separation during mitotic exit. Nat Commun 6, 10076 (2015).

403 37. Gemble, S. et al. Centromere Dysfunction Compromises Mitotic Spindle Pole Integrity. Curr Biol 29, 3072-3080 e3075 (2019).

405 38. Booth, D.G., Hood, F.E., Prior, I.A. \& Royle, S.J. A TACC3/ch-TOG/clathrin 406 complex stabilises kinetochore fibres by inter-microtubule bridging. $E M B O$ $J 30,906-919$ (2011).

408 39. Nixon, F.M. et al. The mesh is a network of microtubule connectors that stabilizes individual kinetochore fibers of the mitotic spindle. Elife 4 (2015).

411 40. Shaheen, R. et al. Genomic and phenotypic delineation of congenital microcephaly. Genet Med 21, 545-552 (2019). 
413 41. Rauch, A. et al. Mutations in the pericentrin (PCNT) gene cause primordial 414 dwarfism. Science 319, 816-819 (2008).

415 42. Willems, M. et al. Molecular analysis of pericentrin gene (PCNT) in a 416 series of $24 \mathrm{Seckel/microcephalic} \mathrm{osteodysplastic} \mathrm{primordial} \mathrm{dwarfism} \mathrm{type}$ II (MOPD II) families. J Med Genet 47, 797-802 (2010).

418 43. Li, F.F. et al. Identification of two novel critical mutations in PCNT gene resulting in microcephalic osteodysplastic primordial dwarfism type II associated with multiple intracranial aneurysms. Metab Brain Dis 30, 1387-1394 (2015). 


\section{Acknowledgements}

425 We are grateful to Drs. M. Berkseth and G. Mirzaa (Seattle Children's Institute) for providing 426 wild-type and CENPE mutant patient LCLs and K. Rhee (Seoul National University) for 427 providing antibodies against PCNT. We thank Masahide Kikkawa (The University of Tokyo), 428 Masafumi Hirono (Hosei University), and Ken-ichi Wakabayashi (Tokyo Institute of 429 Technology) for critical reading of the manuscript. B.D.D. was supported by NIH grant 430 5R01GM120776-09. M.O. was supported by a JSPS Overseas Research Fellowship.

431

\section{Author contributions}

433 M.O. and B.D.D. designed experiments, and M.O. performed all the experiments.

434 M.O. and B.D.D. wrote the manuscript.

435

436 Competing interests: The authors declare no competing interests. 


\section{Methods}

\section{Cell culture}

hTERT-RPE1 cells were cultured in Dulbecco's Modified Eagle's Medium: Nutrient

442 Mixture F12 (DMEM/F12; Corning, 10-092-CV) supplemented with 10\% fetal bovine serum

443 (FBS) and $50 \mathrm{U} / \mathrm{ml}$ penicillin/streptomycin (P/S; Corning, 30-001-CI). HeLa cells were 444 cultured in DMEM (Corning, 10-013-CV) supplemented with 10\% FBS, 1\% L-Glutamine 445 and $\mathrm{P} / \mathrm{S}(50 \mathrm{U} / \mathrm{ml})$. Control and patient LCLs were cultured in Roswell Park Memorial 446 Institute Medium (RPMI 1640; Corning, 10-040-CV) supplemented with 15\% FBS and P/S 447 (50U/ml). RPE-1 and HeLa cells were obtained from the American Type Culture Collection 448 (ATCC). Wild-type (LR09-293m) and patient (LR05-054a2) LCLs were gifts from Dr. 449 Mirzaa (Seattle Children's Hospital). All cells were cultured at $37^{\circ} \mathrm{C}$ with $5 \% \mathrm{CO}_{2}$ and 450 routinely tested with the Universal Mycoplasma Detection Kit (ATCC, 30-1012K).

\section{Production of CENP-E-AID cell line with CRISPR/Cas9}

For the CENP-E-AID cell line, an sgRNA (5'-TTGGCACGCCTCCTCAGGCA-3')

was sub-cloned into PX458 (Addgene, 48138). The C-terminal region of the CENP-E gene was amplified with the following primers; 5'-TGCCCAAACTGGCCTTGAAC-3', 5'-

SK(-) digested with EcoRV. A template vector which carries full-length AID-3 $\times$

Full-length AID-3×FLAG-P2A-BSD was integrated into the site just before the terminal

460 codon of the sub-cloned CENP-E gene. $250 \mathrm{ng}$ of each of the donor and Cas9-sgRNA vectors were transfected to RPE-1 cells cultured in 6 wells with $1.5 \mu$ of FuGENE® HD (Promega, 
463 days, and biallelic mutations in the clones were validated by PCR, followed by DNA 464 sequencing. OsTIR1 and the puromycin-resistant cassette in pBABE TIR1-9myc (Addgene, 465 64945) were replaced with ARF16-PB1-HA-P2A-OsTIR1 from pMGS46 (Addgene, 126580) 466 and the neomycin-resistant cassette, respectively. Retrovirus was produced with the vector and infected to the CENP-E-AID clones, followed by selection with G418 (500 $\mu \mathrm{g} / \mathrm{ml})$.

\section{Cell cycle synchronization}

To increase the number of mitotic cells, RPE1 cells and LCLs were treated with 3 $\mathrm{mM}$ or $2 \mathrm{mM}$ thymidine (Sigma, T9250) for 22 hours, respectively. The RPE1 cells were released for 8 hours and the LCLs for 5 hours before fixation. For synchronization at late G1

473 phase, RPE-1 cells were treated with $0.4 \mathrm{mM}$ mimosine (Sigma, M0253) for 22 hours.

474 Mimosine was washed out twice with fresh media. For synchronization in mitosis, RPE-1

475 cells were first treated with $3 \mathrm{mM}$ thymidine for 22 hours. After washing out thymidine, the 476 cells were incubated with $5 \mu \mathrm{M}$ paclitaxel (Selleck, S1150) for 12 hours or $100 \mu \mathrm{M}$ 477 monastrol for 9 hours. For inhibition of Plk1 activity, cells synchronized with paclitaxel were 478 treated with 100 nM BI2536 (Selleck, S1109) for 3 hours. For forced cell cycle exit, mitotic 479 cells were treated with $2 \mu \mathrm{M}$ ZM447439 (Selleck, S1103) for 1 hour. 0.5 mM IAA (Sigma, 480 I5148) for CENP-E KO cells or $200 \mathrm{nM}$ GSK923295 (APExBIO, A3450) was added right 481 after release from mimosine or thymidine.

\section{Immunofluorescence microscopy}

RPE-1 cells or LCLs on coverslips were fixed with cold methanol at $-20^{\circ} \mathrm{C}$ for $5 \mathrm{~min}$,

485 washed twice with PBS, and blocked with 3\% donkey serum in $0.1 \%$ Triton X-100/PBS for $48615 \mathrm{~min}$. The coverslips were then incubated with primary antibodies for $120 \mathrm{~min}$ at room 487 temperature. Cells were washed with $0.1 \%$ Triton X-100/PBS three times, and the coverslips 
were incubated with secondary antibodies for $90 \mathrm{~min}$ at room temperature, nuclei were stained with DAPI, and coverslips were mounted with ProLong ${ }^{\mathrm{TM}}$ Diamond Antifade

490 Mountant (Invitrogen, P36961). Cells were observed using an Axiovert 200M (63×, NA 1.4,

491 Zeiss) or an LSM800 (63×, NA 1.4, Zeiss). Maximum intensities in Z-stack images were

492 projected to a single image for figures. Signal intensities were measured after subtracting

493 background intensities using ImageJ. For LCLs, coverslips were coated with $0.1 \%$

494 polyethylenimine (PEI), followed by fibronectin (50 $\mu \mathrm{g} / \mathrm{ml}$; Sigma, F2006). Antibodies used

495 for IF are listed in Supplementary Table 1.

Live-cell imaging

H2B-GFP was introduced into CENP-E-AID cells using retroviral delivery as

499 described above, followed by selection with $5 \mu \mathrm{g} / \mathrm{ml}$ of puromycin. Cells expressing 500 H2B-GFP were seeded onto glass bottom dishes (Greiner, 627870) and treated with $3 \mathrm{mM}$ 501 thymidine for 22 hours. 7 hours after thymidine wash-out, cells were observed using an

502 LSM800 confocal microscope (20×, NA 0.8, Zeiss) operated by Zen software (Zeiss). LCLs 503 were synchronized with $2 \mathrm{mM}$ thymidine for 22 hours. 1 hour after release from thymidine, 504 cells were seeded onto glass bottom dishes coated with PEI, and incubated with $2 \mu \mathrm{M}$ 505 Hoechst33342 for $15 \mathrm{~min}$. After changing media, cells were observed using the same 506 equipment and conditions described above. Z-stack (thickness of a slice was $4 \mu \mathrm{m}$ ) images

507 were acquired every $4 \mathrm{~min}$, and multiple slices were projected to one image for figures using 508 ImageJ. We defined cells as obliquely dividing if the center of segregated chromosomes were 509 in different Z-stacks in the first frame of anaphase.

\section{Immunoprecipitation and western blotting}


$513 \mathrm{mM} \mathrm{NaCl}, 0.5 \%$ NP-40, 2mM EDTA, 2 mM EGTA, $1 \mathrm{mM}$ DTT, $10 \mathrm{mM} \mathrm{NaF,} 50 \mathrm{mM}$

$514 \beta$-glycerophosphate, $5 \%$ glycerol and protease inhibitors) on ice for $15 \mathrm{~min}$, followed by 515 centrifugation at $16,000 \times g$ for $15 \mathrm{~min}$. For immunoprecipitation, supernatants were mixed

516 with ANTI-FLAG ${ }^{\circledR}$ M2 Affinity Gel (Sigma, A2220) and rotated at $4{ }^{\circ} \mathrm{C}$ for 3 hours. The

517 beads were washed with lysis buffer four times, and proteins were eluted with $0.1 \mathrm{M}$

518 Glycine-HCl pH 2.8. In SDS-PAGE, $50 \mu \mathrm{g}$ of lysates were loaded into each lane, and 519 proteins were transferred to nitrocellulose membranes (GE Healthcare, 10600007).

520 Antibodies used for western blots are listed in Supplementary Table 1, and uncropped blots

521 are shown in Extended Data Fig. 5.

\section{FACS analysis and cell proliferation assays}

For FACS analysis, harvested cells were fixed with $70 \%$ ethanol for 5 min at $-20^{\circ} \mathrm{C}$,

\section{$531 \quad$ Statistical analysis}

532 Statistical tests, $n$ values, and the number of times that the measurements were

533 repeated were described in figure legends. 
536 Data supporting the findings of this manuscript are available from the corresponding authors

537 upon reasonable request. Source data for all graphs are available. For LCLs, please contact

538 Dr. G. Mirzaa (Seattle Children's Institute).

539

540

541 


\section{Supplementary Table 1: antibodies used in this study}

\begin{tabular}{|c|c|c|c|}
\hline Antibody & Host & Dilution & Reference \\
\hline$\gamma$-tubulin & Mouse & $1: 2000$ (IF) & Sigma \#T5326 \\
\hline Centrin (20H5) & Mouse & $1: 1000$ (IF) & Merc Millipore \#04-1624 \\
\hline Centrin1 & Rabbit & $1: 400$ (IF) & Proteintech \#12794-1-AP \\
\hline Plk1 (F-8) & Mouse & $1: 200$ (IF) & SantaCruz \#sc-376685 \\
\hline CENP-E (C-5) & Mouse & 1:100 (IF), 1:500 (WB) & SantaCruz \#sc-17783 \\
\hline PCNT & Rabbit & $1: 1000$ (IF) & Covance \#PRB-432C \\
\hline PCNT & Rabbit & 1:20000 (IF), 1:5000 (WB) & Lee and Rhee ,2011 \\
\hline PCNT-p1241 & Rabbit & $1: 400$ (IF) & Lee and Rhee ,2011 \\
\hline $\begin{array}{c}\alpha \text {-tubulin } \\
\text { (B5-1-2) }\end{array}$ & Mouse & $1: 3000$ (IF) & Sigma \#T5168 \\
\hline V5 & Mouse & $1: 1000$ (IF) & Invitrogen \#R960-25 \\
\hline PCM1 & Rabbit & 1:1000 (IF), 1:500 (WB) & SantaCruz \#sc-67204 \\
\hline CyclinB1 (GNS1) & Mouse & 1:1000 (WB) & SantaCruz \#sc-245 \\
\hline$\beta$-actin & Mouse & 1:10000 (WB) & Abcam \#ab6276 \\
\hline
\end{tabular}

543 
Owa and Dynlacht. Fig. 1

a

545

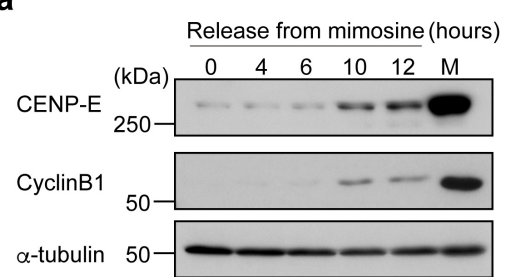

b

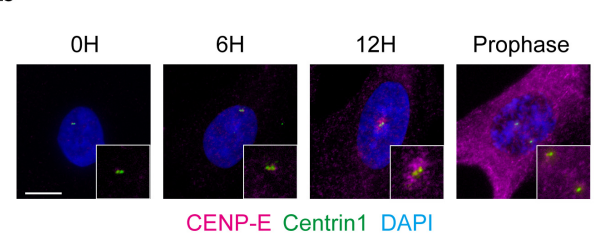

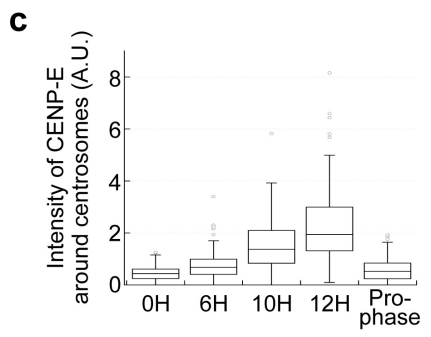

547 Fig. 1 Cytoplasmic CENP-E is recruited around centrosomes in $\mathbf{G}_{\mathbf{2}}$ phase.

548 a, Mimosine synchronized RPE-1 cells were released into fresh media and harvested 0, 4, 6,

54910 and 12 hours after mimosine removal. M phase cells were synchronized with monastrol.

550 Total cell lysates were immuno-blotted with indicated antibodies. b, RPE-1 cells released

551 from mimosine were immuno-stained with antibodies against CENP-E (magenta) and

552 centrin1 (green). Representative pictures 0, 6, and 12 hours after release or in prophase are

553 shown (scale bar $=10 \mu \mathrm{m}$ ). Inset in each panel is a magnified image around centrosomes. c,

554 Signal intensities of CENP-E around centrosomes in (b) were measured and plotted (N=148,

555 two independent experiments; whisker: 95\% confidence interval; box: interquartile; center

556 line: median). 


\section{Owa and Dynlacht. Fig. 2}

a

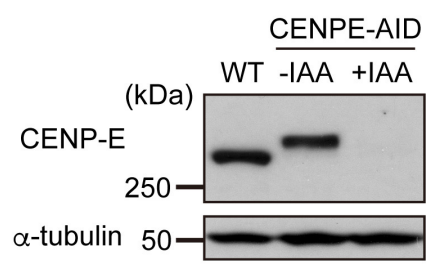

b

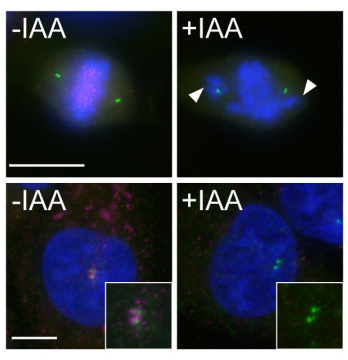

CENP-E Centrin1 DAPI

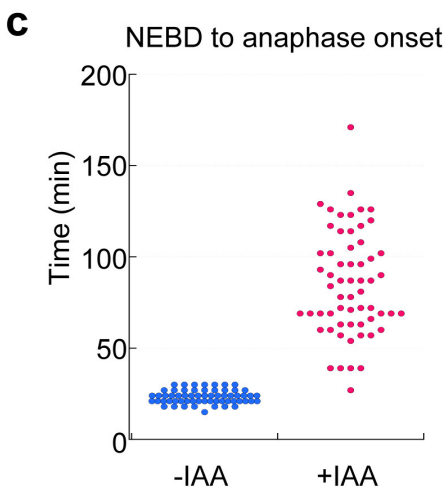

-serum (72 hours) d

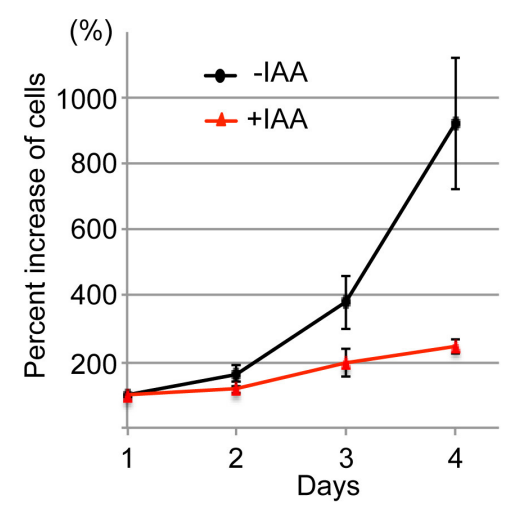

e
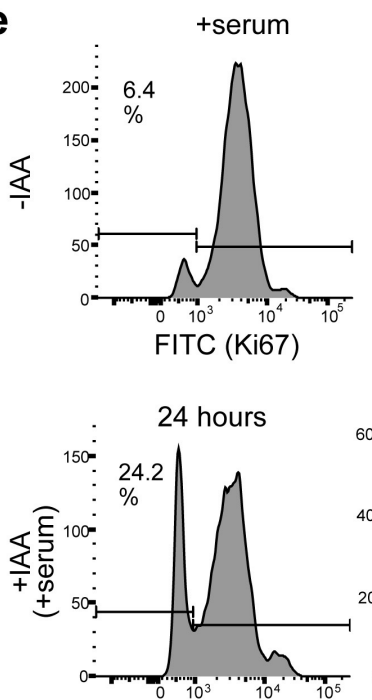
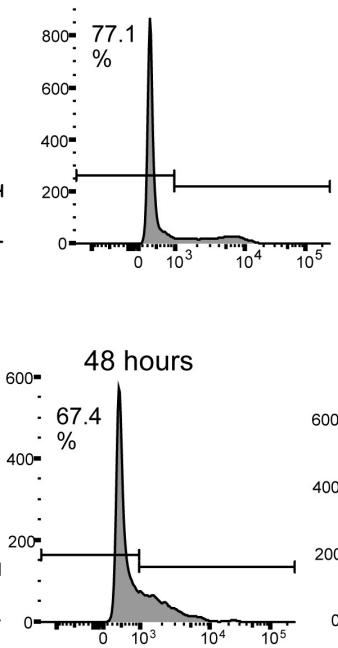

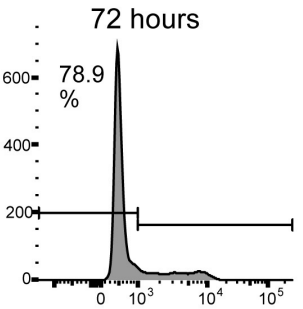

559 Fig. 2 Loss of CENP-E induces cell cycle exit.

560 a, Cell lysates from wild-type RPE-1 and CENP-E-AID (-IAA: not treated; +IAA: treated

561 with IAA for 1 hour) were immuno-blotted with indicated antibodies. The CENP-E band was

562 shifted up in CENP-E-AID due to addition of AID and $3 \times$ FLAG tags. b, Representative

563 images of CENP-E-AID cells immuno-stained with antibodies against CENP-E (magenta)

564 and centrin1 (green). Kinetochore-specific (top panels) and pericentriolar localization

565 (bottom) of CENP-E diminished after IAA treatment. Arrowheads indicate misaligned

566 chromosomes. Scale bar=10 $\mu \mathrm{m}$. c, Time from NEBD to anaphase onset in CENP-E-AID

567 cells with or without IAA was measured by live cell imaging and plotted $(\mathrm{N}=60$, three

568 independent experiments). $\mathbf{d}$, Cell growth assays in CENP-E-AID cells with or without IAA. 
569 Each experiment commenced with $\sim 10,000$ cells (day1). Percent increases of cells were

570 plotted (three independent experiments). e, Proportions of cells that exited from the cell cycle

571 were analyzed by FACS with Ki67-FITC staining. Asynchronous or serum starved

572 CENP-E-AID cells without IAA were used for positive or negative controls, respectively.

573 Time on the histograms indicates duration of IAA treatment.

574

575 
Owa and Dynlacht Fig. 3

a
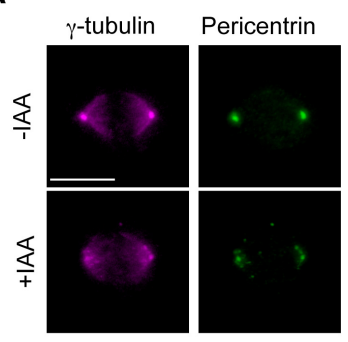

b

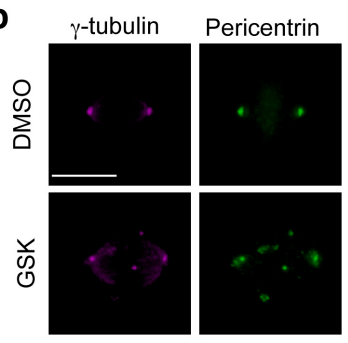

c

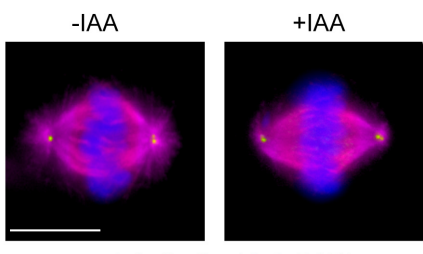

a-tubulin Centrin1 DAPI
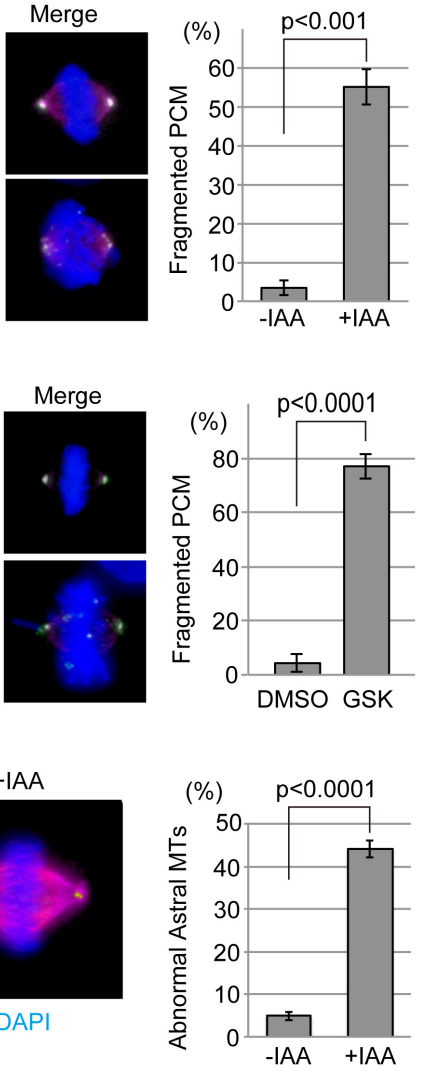

d

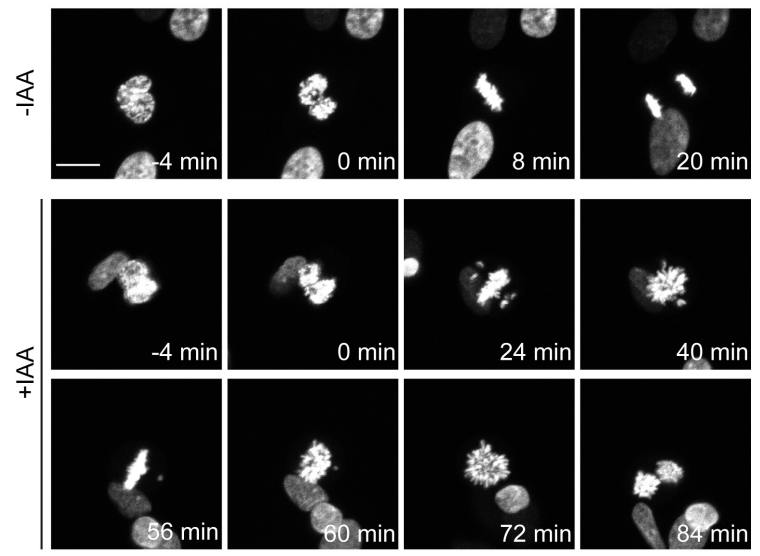

e

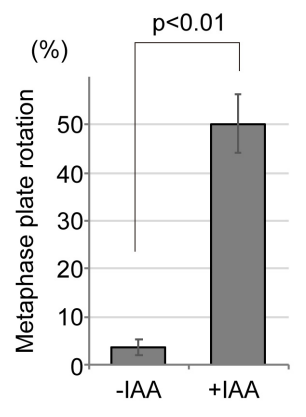

f

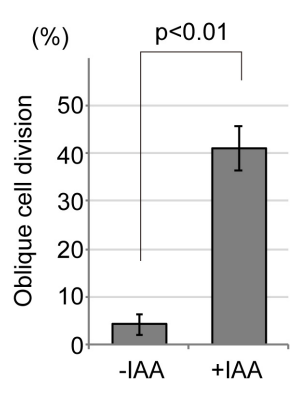

577 Fig. 3 Loss of CENP-E leads to PCM fragmentation and oblique cell divisions

578 a,b, Synchronized CENP-E-AID (a) or wild-type RPE-1 (b) cells with a single thymidine

579 block were released into fresh media for 8 hours with or without indicated drugs. The cells

580 were then fixed and co-immunostained with antibodies against $\gamma$-tubulin (magenta) and

581 PCNT (green). Representative images of metaphase cells in each sample are shown (scale

582 bar $=10 \mu \mathrm{m})$. Percentages of metaphase cells with fragmented PCM are compared in the bar

583 graph ( $>100$ cells in total from three independent experiments; error bars: SD). c,

584 CENP-E-AID cells treated as in panel (a) were co-immunostained with antibodies against

$585 \alpha$-tubulin (magenta) and centrin1 (green). Representative images of metaphase cells in each

586 sample are shown (scale bar=10 $\mu \mathrm{m}$ ). Percentages of metaphase cells with absent astral MTs

587 are compared in the bar graph ( $>100$ cells in total from three independent experiments; error

588 bar: SD). d, Live-cell imaging of CENP-E-AID cells stably expressing H2B-GFP. 
589 Representative time-course images of control (-IAA) or CENP-E KO cells (+IAA) are shown

590 (scale bar $=10 \mu \mathrm{m}$ ). e, Percentages of cells in which the metaphase plate was rotated within 1

591 hour after NEBD are shown in the bar graph ( $>100$ cells in total from three independent

592 experiments; error bars: SD). f, Percentages of oblique cell divisions (see methods for 593 definition) are shown in the bar graph ( $>100$ cells in total from three independent 594 experiments; error bars: SD). p-values were calculated by unpaired $t$-tests. 


\section{Owa and Dynlacht. Fig. 4}

a

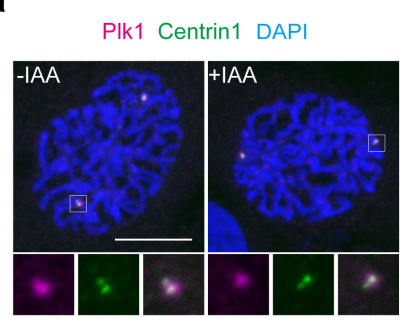

e

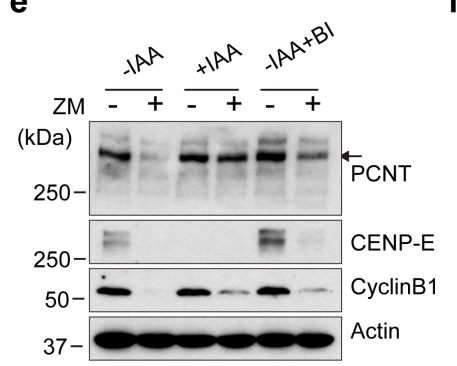

597

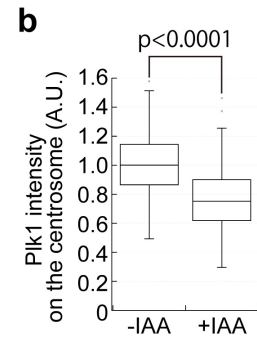

c

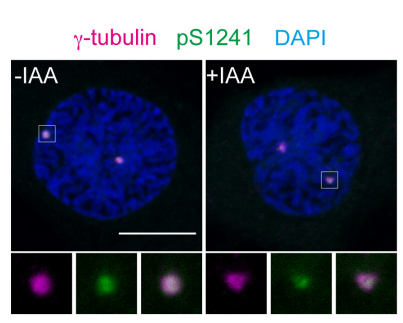

d

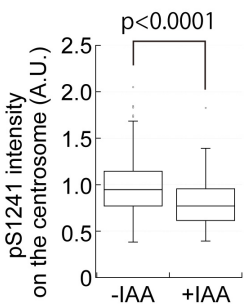

f

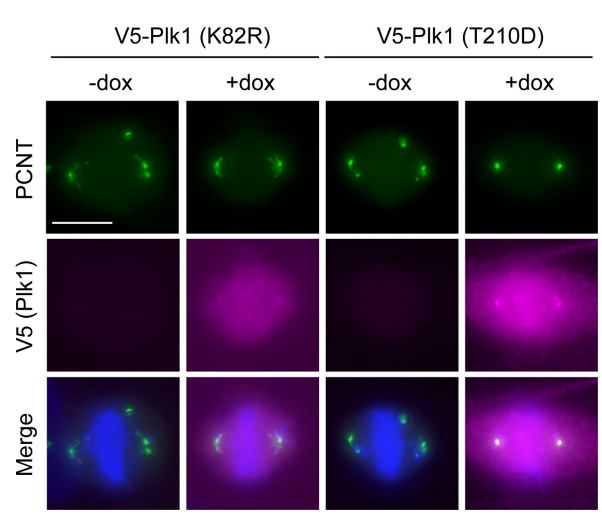

g

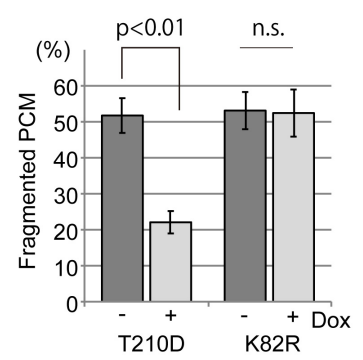

Fig. 4 PCM1 fragmentation in CENP-E KO results from reductions in centrosomal

PIk1 levels and aberrant phosphorylation of PCNT. a,b, Representative images for IAA-treated or un-treated CENP-E-AID cells in prophase co-immunostained with antibodies against Plk1 (magenta) and centrin1 (green) (a, Scale bar=10 $\mu \mathrm{m})$. The area enclosed by the square in each image is magnified and shown under the panel. Relative Plk1 intensities on the centrosome in prophase cells were plotted in $(\mathbf{b})(\mathrm{N}=90$, three independent experiments; whisker: 95\% confidence interval; box: interquartile; center line: median). c,d, Representative images for IAA-treated or untreated CENP-E-AID cells in prophase co-immunostained with antibodies against $\gamma$-tubulin (magenta) and PCNT-pS1241 (green) (c, Scale bar $=10 \mu \mathrm{m})$. The area enclosed by the square in each image is magnified and shown under the panel. Relative PCNT-pS1241 intensities on the centrosome in prophase cells were plotted in (d) $(\mathrm{N}=90$, three independent experiments; whisker: 95\% confidence interval; box: interquartile; center line: median). e, Control (-IAA), CENP-E KO (+IAA), and Plk1 inhibited (-IAA+BI) cells synchronized with $100 \mathrm{nM}$ paclitaxel were shaken off from plates 
612 and forced to exit from mitosis with $2 \mu \mathrm{M}$ ZM44739 for 1 hour. Cell lysates were

613 immuno-blotted with indicated antibodies. The arrow indicates full-length PCNT. f,g,

614 CENP-E-AID cell lines stably expressing doxycycline (dox)-inducible V5-tagged Plk1

615 (T210D: constitutively active; K82R: catalytically inactive) were synchronized with

616 thymidine for 22 hours, and released for 8 hours. The cells were stained with antibodies

617 against V5-tag (magenta) and PCNT (green). In +dox samples, $1 \mu \mathrm{g} / \mathrm{ml}$ dox was maintained

618 throughout the experiments. Representative images for metaphase cells in each sample are

619 shown (f, scale bar=10 $\mu \mathrm{m})$. Percentages of metaphase cells with fragmented PCM are

620 compared in (g) (>100 cells in total from three independent experiments; error bars: SD).

621 p-values were calculated by Mann-Whitney $U$ tests $(\mathrm{b}, \mathrm{d})$ or unpaired $t$-tests $(\mathrm{g})$.

622

623 
Owa and Dynlacht. Fig. 5

a

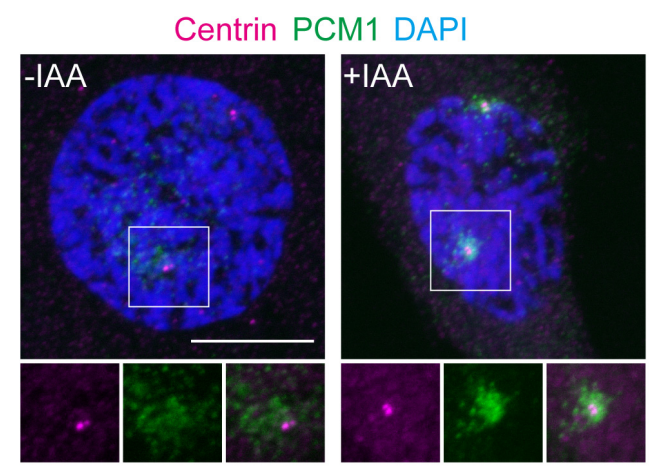

b

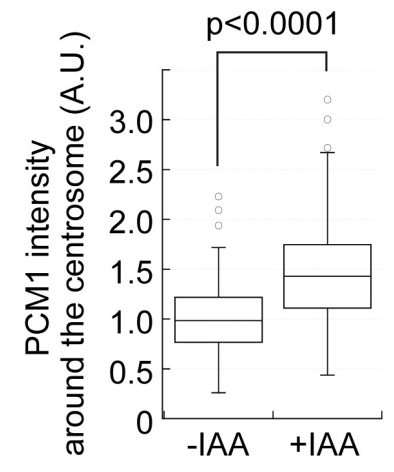

C
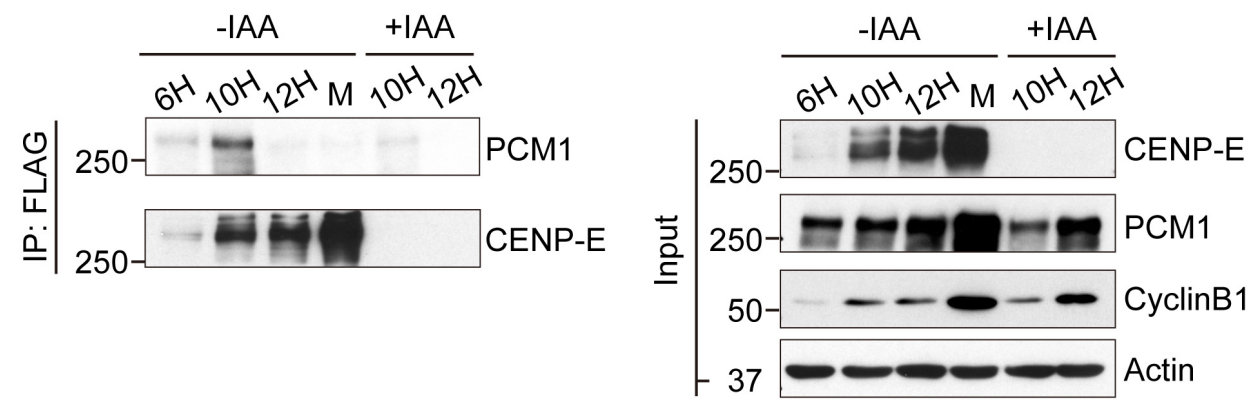

625 Fig. 5 Loss of CENP-E induces accumulation of PCM1 around centrosomes in prophase.

$626 \mathbf{a , b}$, Representative images for IAA treated or untreated CENP-E-AID cells in prophase

627 co-immunostained with antibodies against centrin (magenta) and PCM1 (green) (a, Scale

628 bar $=10 \mu \mathrm{m})$. The area enclosed by the square in each image is magnified and shown under

629 the panel. Relative PCM1 intensities around the centrosome in prophase cells were plotted in

630 (b) $(\mathrm{N}=90$, three independent experiments; whisker: 95\% confidence interval; box:

631 interquartile; center line: median). The p-value was calculated by a Mann-Whitney $U$ test. c,

632 Cell lysates from mimosine $(6 \mathrm{~h}, 10 \mathrm{~h}$, and $12 \mathrm{~h}$, indicating hours after release) or monastrol

633 (M) synchronized CENP-E-AID cells were immunoprecipitated with FLAG-M2 beads. 10h

634 and $12 \mathrm{~h}(+\mathrm{IAA})$ samples were for negative controls. The precipitates were immuno-blotted

635 with anti-CENP-E and anti-PCM1 antibodies (left). Inputs in each sample were also blotted

636 with indicated antibodies (right). 


\section{Owa and Dynlacht Fig. 6}

a

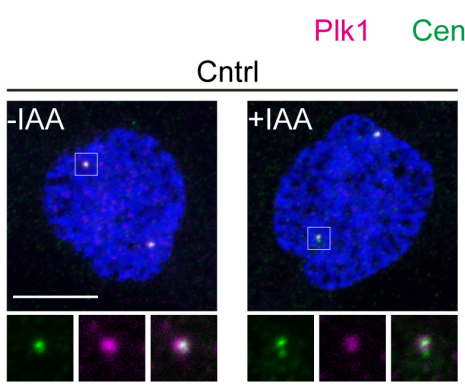

C

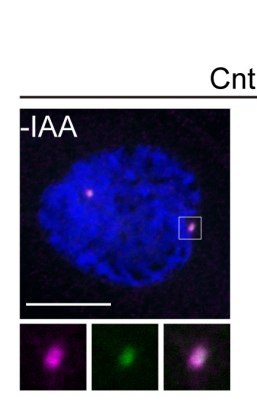

e

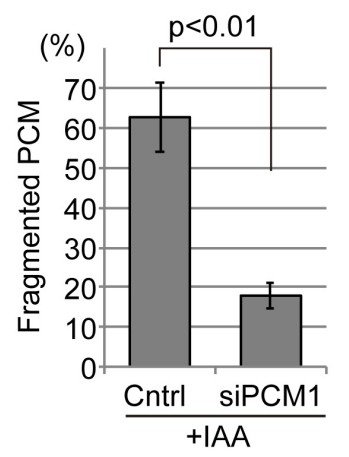

$\gamma$-tubulin pS1241 DAPI
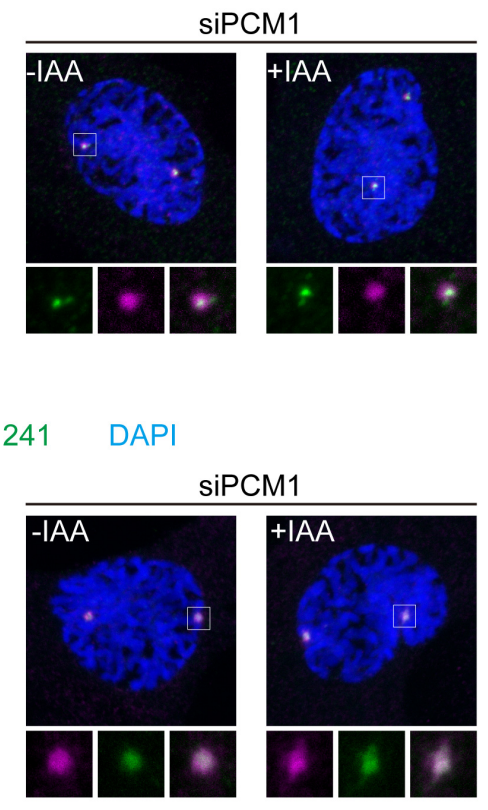

f b

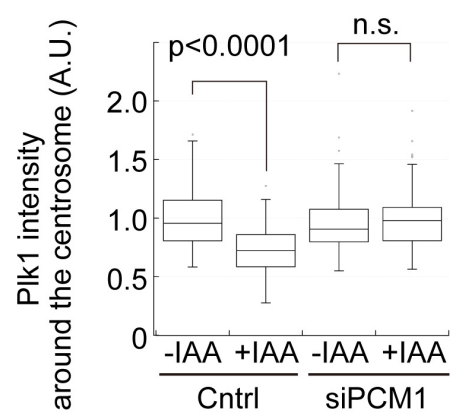

d

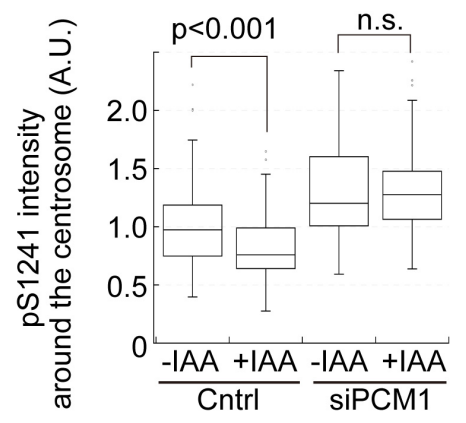

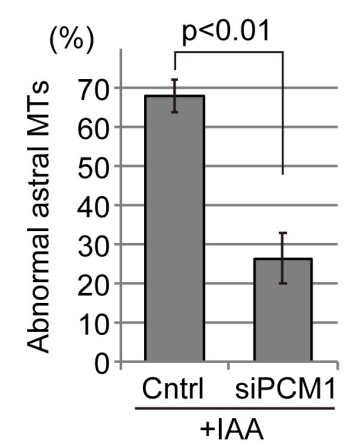

638 Fig. 6 Accumulation of PCM1 around centrosomes perturbs centrosomal Plk1

639 recruitment and PCNT phosphorylation in CENP-E KO. a, CENP-E-AID cells were

640 synchronized with thymidine for 22 hours. In the first 8 hours of the synchronization, cells

641 were treated with control siRNA or siPCM1. The cells were then released for 8 hours with or

642 without IAA, fixed, and co-immunostained with antibodies against Plk1 (magenta) and

643 centrin1 (green). Representative images for prophase cells in each sample are shown. The

644 area enclosed by the square in each image is magnified and shown under the panel. Scale

645 bar $=10 \mu \mathrm{m} . \mathbf{b}$, Box plots of relative Plk1 intensities on centrosomes in prophase cells $(\mathrm{N}=90$,

646 three independent experiments; whisker: 95\% confidence interval; box: interquartile; center 
647 line: median). c,d, Samples prepared as (a) were co-immunostained with antibodies against

$648 \gamma$-tubulin (magenta) and PCNT-pS1241 (green). Representative images for prophase cells in

649 each sample are shown (c, Scale bar=10 $\mu \mathrm{m})$. The area enclosed by the square in each image

650 is magnified and shown under the panel. Relative PCNT-pS1241 intensities on the

651 centrosome in prophase cells were plotted in (d) $(\mathrm{N}=90$, three independent experiments;

652 whisker: 95\% confidence interval; box: interquartile; center line: median). e,f, CENP-E-AID

653 cells were synchronized with siRNA treatments as (a). The cells were then released for 8

654 hours with IAA, and percentages of PCM fragmentation (e) or abnormal astral MTs (f) were

655 calculated as in Fig. 3a and c (>100 cells in total from three independent experiments; error

656 bars: SD). p-values were calculated by Mann-Whitney $U$ tests (b, d) or unpaired $t$-tests (e, f). 
Owa and Dynlacht. Fig. 7

a

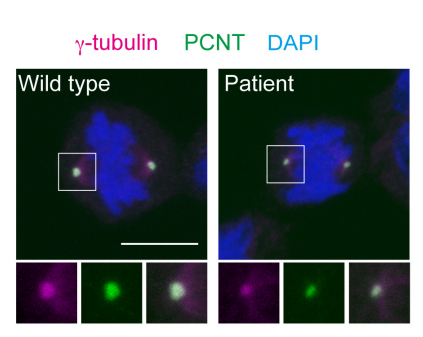

C

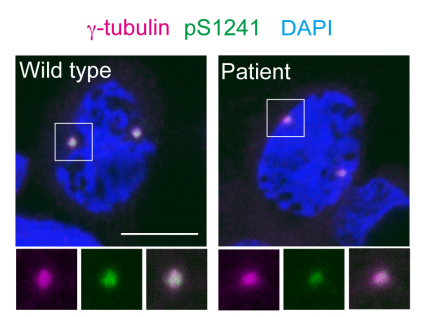

b

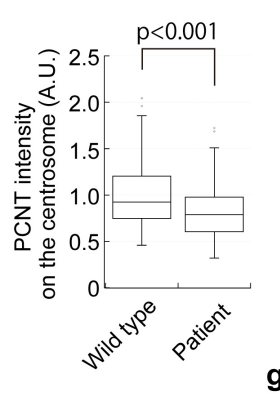

d

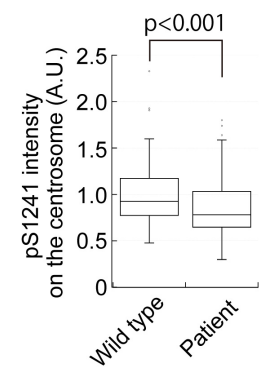

g
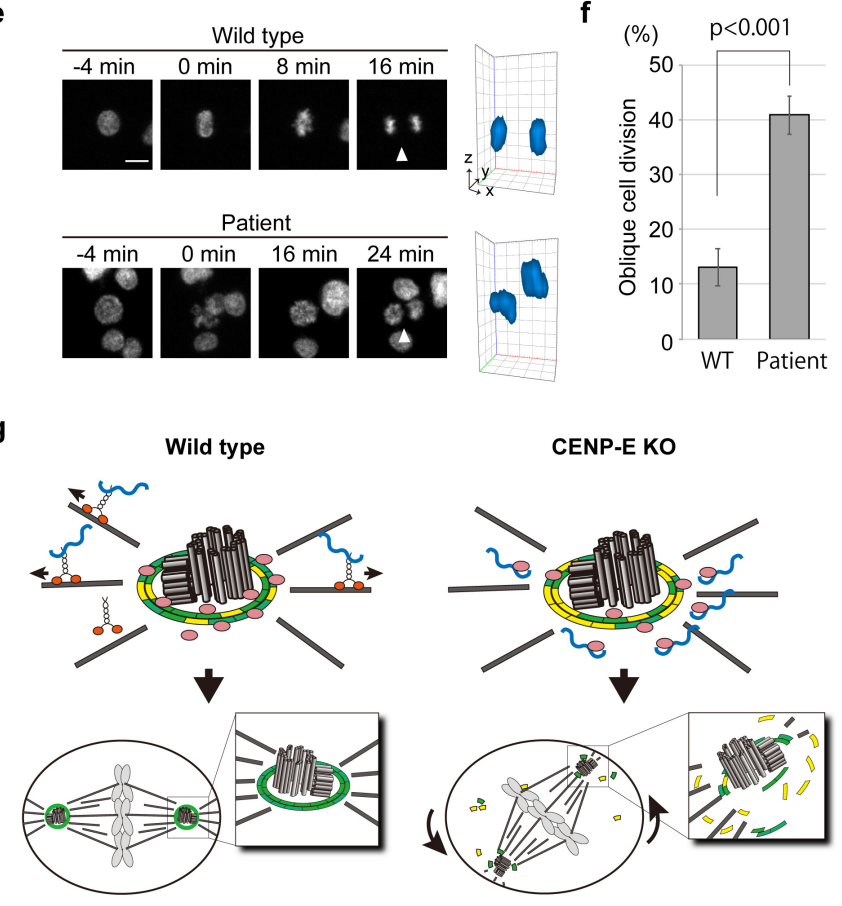

CENP-E KO

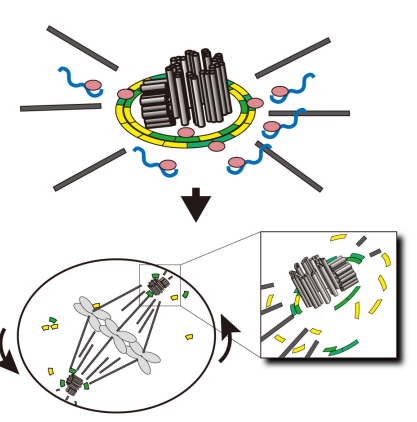

CENP-E $\sim$ PCM1 $\bigcirc$ Plk1 MT

$\triangle$ Un-phosphorylated PCM $\rightleftharpoons$ Phosphorylated PCM

660 Fig. 7 LCLs from a microcephalic patient with CENP-E mutations show PCM defects

664 (a, Scale bar=10 $\mu \mathrm{m}$ ). The area enclosed by the square in each image is magnified and shown 665 under the panel. Relative PCNT intensities on the centrosome in metaphase cells were plotted 666 in (b) ( $\mathrm{N}=90$, three independent experiments; whisker: 95\% confidence interval; box: 667 interquartile; center line: median). c,d, Representative images for prophase LCLs 668 co-immunostained with antibodies against $\gamma$-tubulin (magenta) and PCNT-pS1241 (green) (c,

669 Scale bar=10 $\mu \mathrm{m})$. Relative PCNT-pS1241 intensities on the centrosome in metaphase cells 670 were plotted in $(\mathbf{d})(\mathrm{N}=90$, three independent experiments; whisker: 95\% confidence interval;

671 box: interquartile; center line: median). e, Live-cell imaging of wild-type and patient LCLs.

672 DNA was visualized with Hoechst 33342. Representative time-course images of wild-type 
673 (top) or patient (bottom) LCLs are shown (scale bar=10 $\mu \mathrm{m}$ ). 3D reconstructions in the last

674 time points (viewed from arrowheads) are also shown next to the images. f, Percentages of

675 oblique cell divisions observed in the live-cell imaging (see methods for definition) were

676 compared in the bar graph $(>100$ cells in total from three independent experiments; error

677 bars: SD). p-values were calculated by Mann-Whitney $U$ tests (b, d) or an unpaired $t$-test (f).

678 g, A model summarizing results in this study. See text for details.

679

680 
Owa and Dynlacht. Extended Data Fig. 1

a

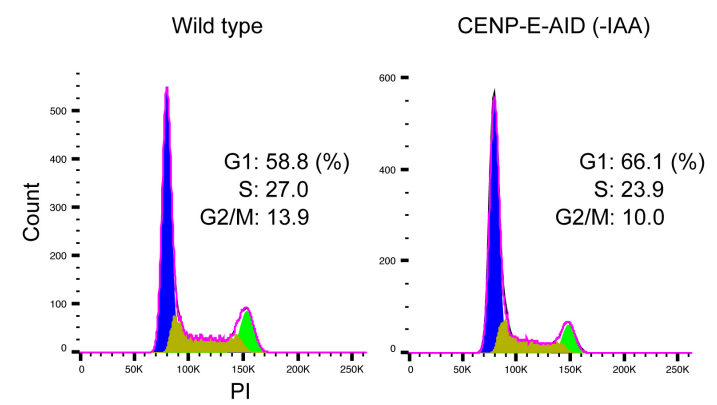

b

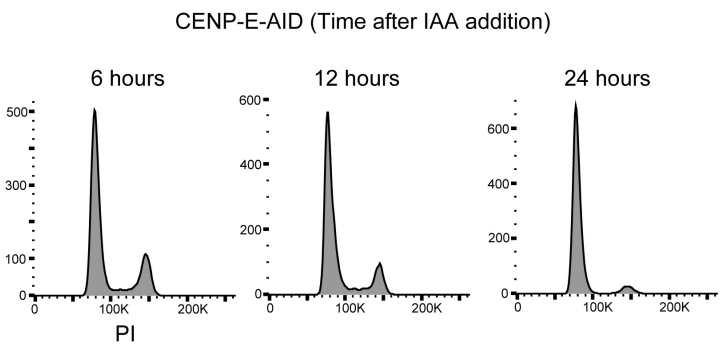

681

682 Extended Data Fig. 1 Further characterization of the CENP-E-AID cell line.

683 a, Cell cycle profiles of asynchronous wild-type cells and asynchronous CENP-E-AID cells

684 without IAA treatment. DNA content was analyzed by FACS using propidium iodide (PI)

685 staining. b, Cell cycle profiles of CENP-E-AID cells treated with IAA. Asynchronous

686 CENP-E-AID cells were treated with IAA for indicated hours and analyzed by FACS (PI).

687 Time on the histograms indicates duration of IAA treatment.

688

689 


\section{Owa and Dynlacht Extended Data Fig. 2}

a

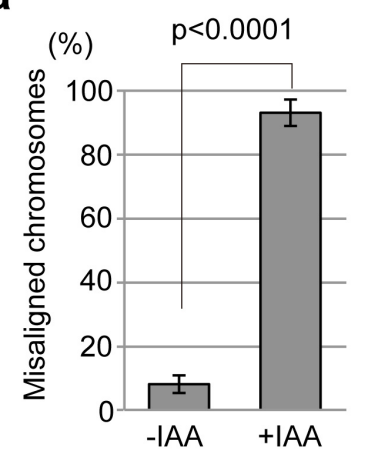

d

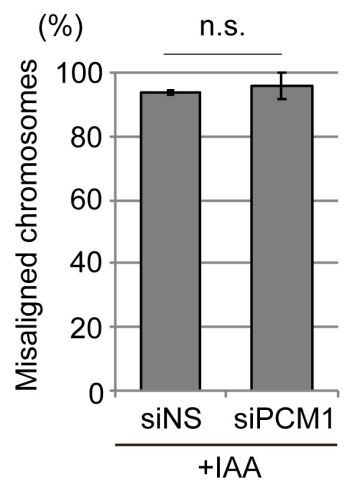

b

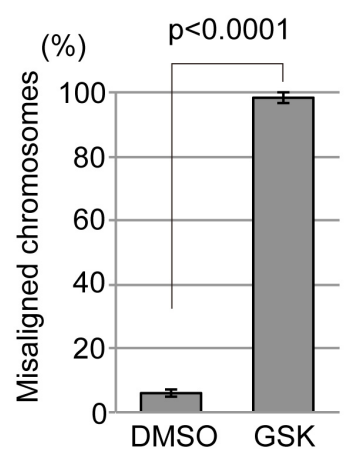

C

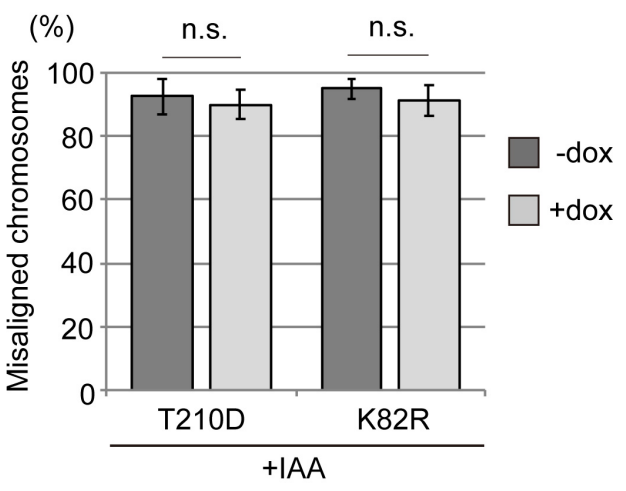

690

\section{Extended Data Fig. 2 Percentages of misaligned chromosomes.}

692 Percentages of cells that possess one or more misaligned chromosomes around the spindle

693 pole. Error bars: SD. a, CENP-E-AID cells with or without IAA; b, wild-type cells treated

694 with DMSO or GSK; c, CENP-E-AID cells stably expressing doxycycline (dox)-inducible

695 V5-tagged Plk1 (T210D or K82R), treated with IAA only or IAA and dox; d, CENP-E-AID

696 cells treated with control siRNA and IAA or siPCM1 and IAA. 


\section{Owa and Dynlacht Extended Data Fig. 3}

a

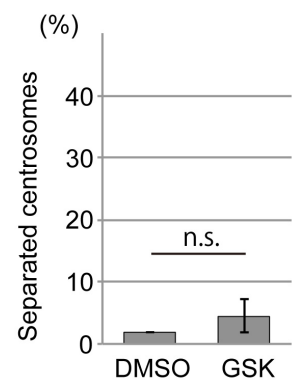

b
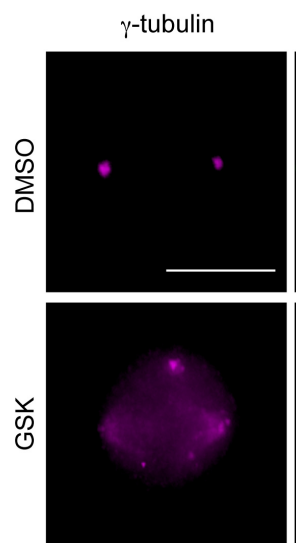

Centrin1
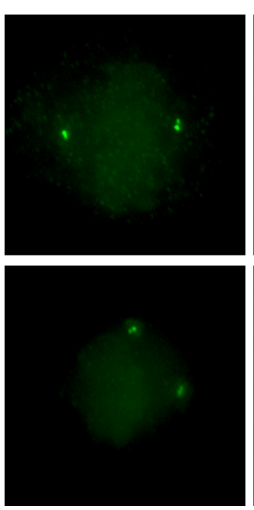

Merge

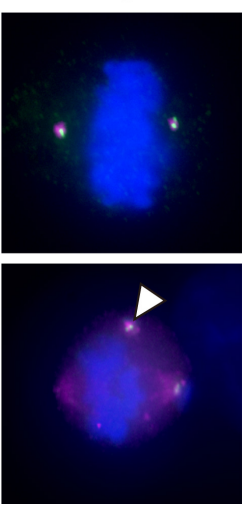

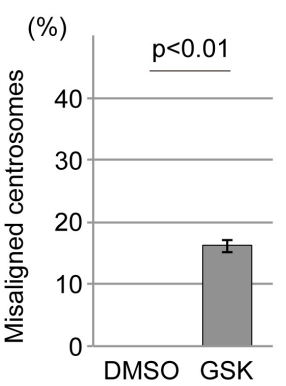

700 Extended Data Fig. 3 Centriole detachment from the spindle pole in CENP-E KO.

701 Wild-type RPE-1 cells were synchronized with thymidine and released for 8 hours with

702 DMSO or GSK. The cells were co-immuno-stained with antibodies against $\gamma$-tubulin

703 (magenta) and centrin1 (green). a, Percentages of precocious centriole disengagement ( $>100$

704 cells in total from three independent experiments; error bars: SD). b, Representative images

705 for normal (top, DMSO) or misaligned centrioles (bottom, GSK). The arrowhead indicates

706 centrioles detached from the spindle pole. Percentages of centrioles detached from the spindle

707 pole are shown in the bar graph $(>100$ cells in total from three independent experiments; error

708 bars: SD). p-values were calculated by unpaired $t$-tests.

709 


\section{Owa and Dynlacht. Extended Data Fig. 5}

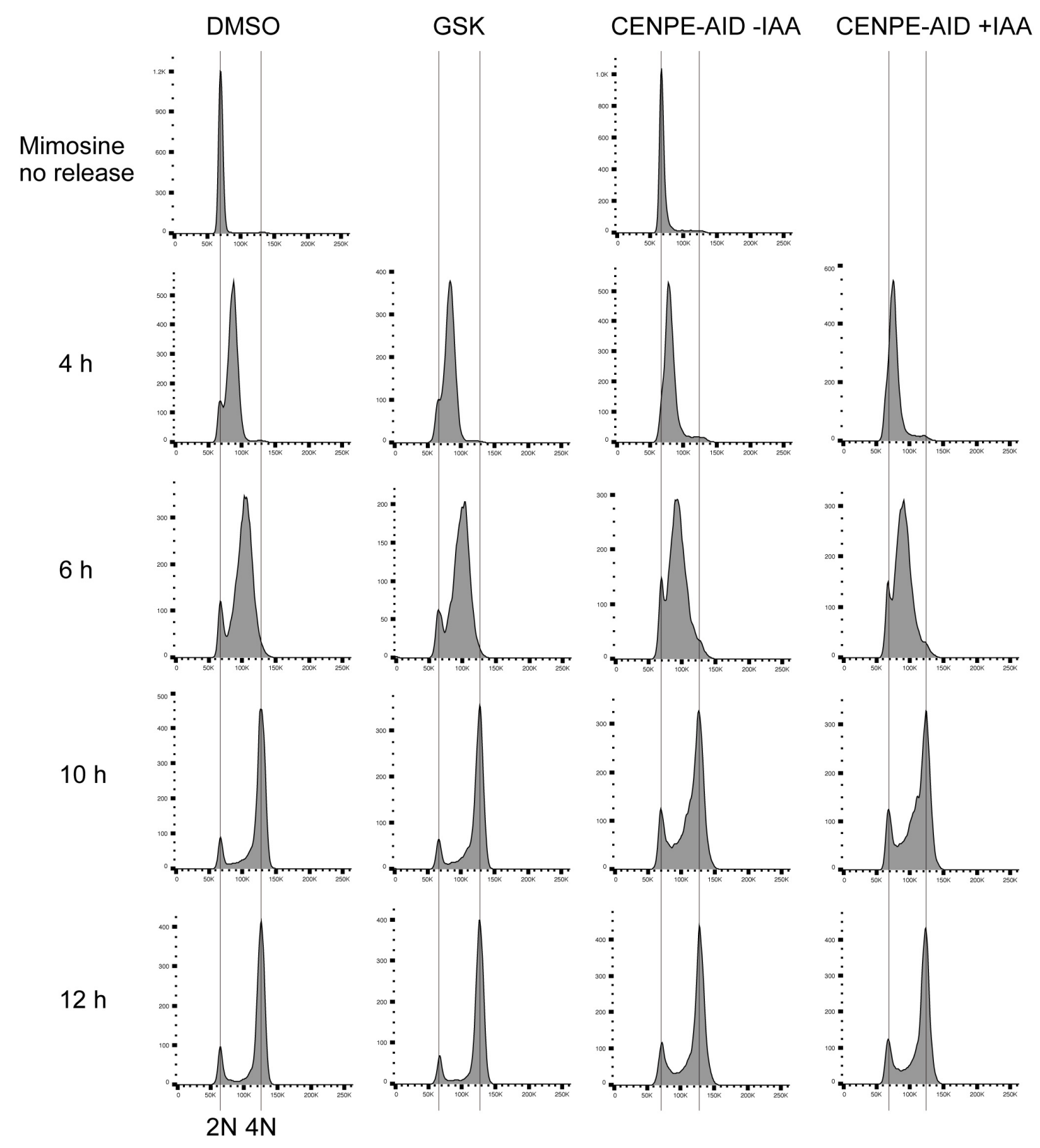

712 Extended Data Fig. 4 Cell cycle profiles of mimosine-synchronized cells.

713 Mimosine synchronized wild-type RPE-1 cells or CENP-E-AID cells treated with indicated

714 drugs were released for 4, 6, 10 and 12 hours and analyzed by flow cytometry. Histograms of

715 DNA content at each time point are shown $\left(2 \mathrm{~N}: \mathrm{G}_{1} ; 4 \mathrm{~N}: \mathrm{G}_{2} / \mathrm{M}\right)$ 


\section{Owa and Dynlacht Extended Data Fig. 5}

Fig. 1a

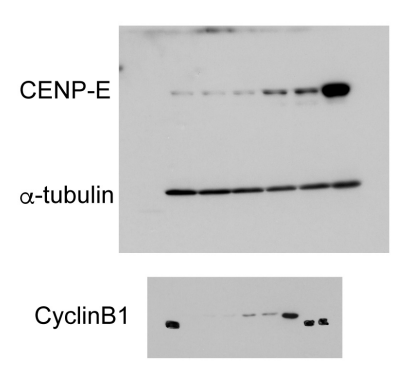

Fig. 3a

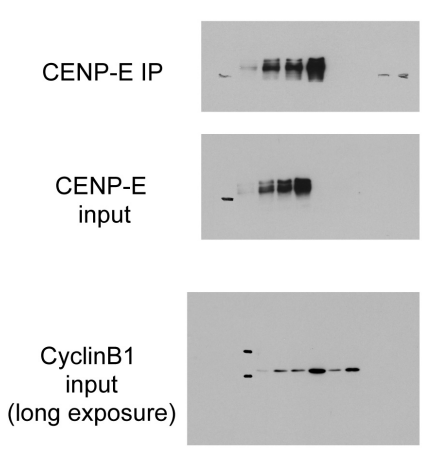

717

718 Extended Data Fig. 5 Uncropped images of western blots.
Fig. 2e

Fig. 1c

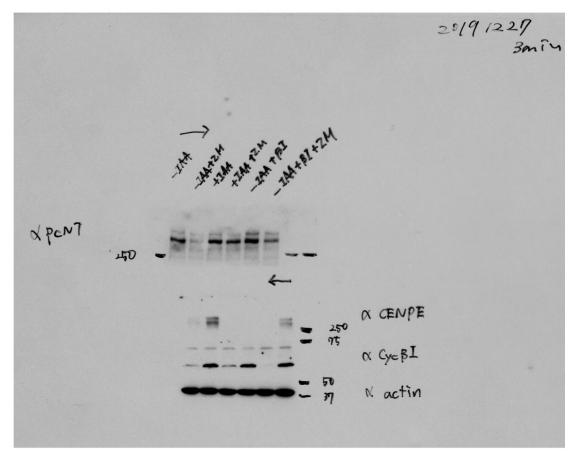

